\title{
Retrograde-Assisted Percutaneous Cystolitholapaxy Versus Transurethral Cystolithotripsy With Holmium-YAG Laser: A Retrospective Study
}

\author{
Shahrokh Sakhaei ${ }^{1}$, Morteza Fallah-Karkan ${ }^{2,3}$, Mohammadreza Razzaghi ${ }^{2,4}$, Babak Kazemzadeh Azad ${ }^{5^{*}}$, \\ Fereshteh Aliakbari ${ }^{5}$ \\ ${ }^{1}$ Urology Department, Kermanshah University of Medical Sciences, Kermanshah, Iran \\ ${ }^{2}$ Laser Application in Medical Science Research Center, Shahid Beheshti University of Medical Sciences, Tehran, Iran \\ ${ }^{3}$ Urology and Nephrology Research Center, Shahid Beheshti University of Medical Sciences, Tehran, Iran \\ ${ }^{4}$ Urology Department, Shohada-e-Tajrish hospital, Shahid Beheshti University of Medical Sciences, Tehran, Iran \\ ${ }^{5}$ Infertility \& Reproductive Health Research Center, Shahid Beheshti University of Medical Sciences, Tehran, Iran
}

\section{*Correspondence to \\ Babak Kazemzadeh-Azad, Infertility \& Reproductive Health Research Center, Shahid Beheshti University of Medical Sciences, Tehran, Iran. \\ Tel/Fax: +98 2122712234 \\ Mob: +98 9111863352; \\ Email: bab1412000@yahoo.com}

Published online December 1 2019

\begin{abstract}
Introduction: The retrograde approach is a modification that makes the percutaneous cystolitholapaxy (PCCL) a more trendy method, especially in operating rooms with limited facilities. The transurethral approach for bladder calculi lithotripsy by a laser has become popular among urologists. In this study, we investigate the feasibility and safety of retrograde assisted access for PCCL in comparison with transurethral cystolithotripsy by the holmium-YAG laser (Ho: YAG).

Methods: According to the type of intervention, the patients were stratified to two matched groups. In the retrograde-assisted percutaneous cystolitholapaxy (RPCCL) group, a Benique was conducted through the urethra into the bladder; palpating the suprapubic region, an about $1.5 \mathrm{~cm}$ incision was done over the tip, then an Amplatz sheath was placed over it, treading into the bladder; further cystolitholapaxy was done by a routine order. In transurethral Ho: YAG laser lithotripsy (TULL) via $200 \mu \mathrm{m}$ fiber vaporize the stone.

Results: A total of 124 male patients with the mean age of $50.33 \pm 9.64$ years and the average stone burden of $3.35 \pm 1.07 \mathrm{~cm}$ were included in the study. The most common cause of vesical calculi was spinal cord injury. Statistically significant differences were found in terms of the mean operation time in favor of the RPCCL group $(P \leq 0.05)$ and the mean hospital stay in favor of the TULL group $(P \leq 0.05)$. The stone-free rate $(S F R)$ was $100 \%$ in both methods after a onemonth follow-up. None of the interventions changed to open surgery. There were not any major complications in both methods.

Conclusion: RPCCL is a safe and effective method in bladder stone treatment and is applicable in medical centres without Ho: YAG equipment.

Keywords: Bladder stone; Bladder calculi; Percutaneous cystolitholapaxy; Transurethral cystolitholapaxy; Holmium-YAG laser.
\end{abstract}

\section{Introduction}

Bladder calculi account for $5 \%$ of urinary tract calculi in the western world and are typically associated with lower urinary tract obstruction in older men. ${ }^{1}$ Various therapeutic modalities have been applied throughout the decades such as open suprapubic cystotomy, transurethral cystolitholapaxy and percutaneous cystolitholapaxy (PCCL) by lasers and/or Lithocast., ${ }^{2,3}$ Despite all these therapies, the procedure of choice must be both rather noninvasive and quick. ${ }^{4-6}$ The Ho: YAG laser has been great evolution in the management of urinary lithiasis. The smallest stone particles and decreases in the likelihood of tissue destruction are achieved by the Ho: YAG laser when compared with other modalities of lithotripsy. ${ }^{7}$ However, the high cost of the laser and the lack of access to all the centers have limited its use. ${ }^{3}$

In recent years, percutaneous techniques for treating bladder calculi in both children and adults have appeared. ${ }^{8,9}$ PCCL is an established technique with low morbidity and low complication rates for large-burden vesical stones. It has been performed safely for bladder stones measuring $<5 \mathrm{~cm} .{ }^{10}$ It provides a high stone-free rate (SFR) with a less scar and morbidity in comparison with an open approach. ${ }^{11}$ Also, PCCL Can be accompanied

Please cite this article as follows: Sakhaei S, Fallah-Karkan M, Razzaghi M, Kazemzadeh Azad B, Aliakbari F. Retrograde-assisted percutaneous cystolitholapaxy versus transurethral cystolithotripsy with holmium-YAG laser: a retrospective study. J Lasers Med Sci. 2019;10(suppl 1):S54-S58. doi:10.15171/jlms.2019.S10. 
by complications such as hematuria easily treated with non-surgical measures. ${ }^{12}$

In this study, we evaluate the safety and efficacy of a new modification in access to the bladder in percutaneous cystolithotomy for treatment of adult vesical stones in comparison with transurethral cystolithotripsy with the Ho: YAG laser.

\section{Methods}

Study Population

In a retrospective investigation from May 2013 to September 2018, 65 patients underwent PCCL with the retrograde approach in Imam Reza hospital, Kermanshah Universityof Medical Sciences, Kermanshah, Iran; and 57 cases in Shohada-e-Tajrish hospital, Shahid Beheshti Universityof Medical Sciences, Tehran, Iran underwent TULL. The patients were matched in 2 groups.

\section{Inclusion and Exclusion Criteria}

122 consecutive male patients with large bladder stones were enrolled in the investigation. The subjects with the history of bladder malignancy, pelvic radiotherapy, radical prostatectomy, urethral stenosis/stricture or disruption, and urinary infection and the patients who needed prostatectomy or TURP due to significant high prostate volume and body mass index $\geq 30$ were excluded.

\section{Surgical Technique}

Kidneys, ureters, bladder and prostate ultrasound were done in all patients. Preoperative laboratory tests included serum creatinine and hemoglobin measurements, platelet counts, coagulation screen tests and urine cultures. A single dose of intravenous ciprofloxacin $400 \mathrm{mg}$ was administered as antibiotic prophylaxis.

\section{PCCL With Retrograde Assistance}

Under spinal anesthesia and in the lithotomy position, a Lowsley sound or an appropriate Benique was passed through the urethra into the bladder. Palpating the suprapubic region, an about $1.5 \mathrm{~cm}$ incision was made over the tip of the sound or Benique.

The incision was deepened until the tip of the sound appeared, then a 28 or $30 \mathrm{~F}$ Amplatz sheath was placed over the tip of the sound, treading into the bladder over the sound. Advancing the Amplatz sheath into the bladder, the Benique was emitted from the urethra (Figure 1). A zero-degree rigid nephroscope was advanced into the bladder, and the stones were fragmented using the Swiss Lithoclast (EMS, Switzerland) and the fragmented stones were removed by forceps. The Incision was closed by 1 or 2 far and near suture and a 20 Fr urethral Foley catheter placed for 1-2 days. The patients would be discharged after one-day hospitalization if there were any complications.

\section{TULL}

After local anesthesia by $15 \mathrm{cc}$ of $2 \%$ lidocaine gel plus
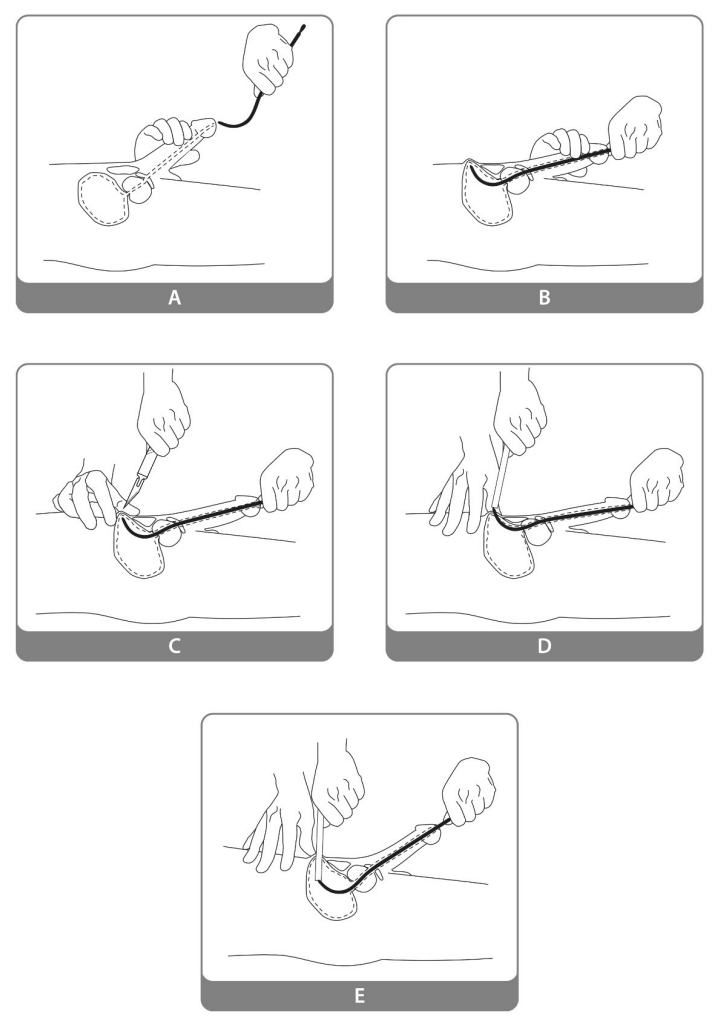

Figure 1. Retrograde PCCL Step by Step.

5 cc of a 1\% lidocaine ampoule was instilled, penile clamps were used for 10 minutes. Then a semi-rigid 19 Fr Cystoscope (Karl Storz Inc., Germany) was introduced to the bladder and the Ho: YAG laser (Iranian National Laser Center Science \& Technology, Iran) via $200 \mu \mathrm{m}$ micrometers end-fire fibers was applied with the power of 5 to $10 \mathrm{~W}$ and frequency of $10-15 \mathrm{~Hz}$ to vaporize the stone. Stone fragments were evacuated by irrigation, suction and grasper and at the end of the procedure, 18 or $20 \mathrm{Fr}$ indwelling urethral catheter was placed. All the patients were discharged from hospital on the operation day if there were any complications. At the time of discharge, the urethral catheters were removed. The use of an oral antibiotic (ciprofloxacin $500 \mathrm{mg} / \mathrm{Bid}$ ) continued for 24 hours.

\section{Study Outcome}

The duration of admission, the operative time, the SFR, hematuria which lasts more than 2 days after surgery, and other probable complications were recorded. Follow-ups were done 1 week and 1 month later.

\section{Data Analysis}

We used SPSS software version 19.0 for data analysis in all steps. The $P<0.05$ was considered as the statistical significance level. The qualitative data was presented with frequency and percentage and their analysis was done with the chi-square test. Descriptive statistics (mean \pm 
standard deviation) and Student $t$ test was used show and analyzes the quantitative outcomes.

\section{Results}

Demographic and clinical characteristics among the 2 groups are illustrated in Table 1 . The groups were similar in the age, body mass index, mean size of stones, and number of stones.

Statistically significant differences were found in terms of the mean operation time in favor of the retrogradeassisted percutaneous cystolitholapaxy (RPCCL) group $(P \leq 0.05)$ and the mean hospital stay in favor of the TULL group $(P \leq 0.05)$.

The SFR was $100 \%$ in both methods after a one-month follow-up. None of the interventions changed to open surgery.

There were no major complications (grade III-V Clavien-Dindo classification), including overadvancement, urinary leakage, bladder perforation and surgical site infection. No electrolyte changes were observed. Visible Hematuria, also known as the gross hematuria which lasts at least 2 days, was seen in 5 cases in RPCCL arm and it was solved with bladder irrigation. Urinary retention happened in 2 cases in RPCCL after catheter removal. All of them were $\mathrm{BPH}$ patients who were managed by Nelaton catheter bladder drainage and alphablocker medication. The 17 (11 in TULL; 6 in RPCCL) patients had mild urinary irritative symptoms that were controlled by anticholinergic agents. Three patients in the TULL group and 4 in the RPCCL group were admitted for 2 days due to post-operative fever. Their fever was treated by intravenous antibiotic therapy. In 4 patients of the TULL group, intravenous sedation was performed during the operation. Their operation time lasted for nearly 45 minutes because of the stone diameter $\geq 4 \mathrm{~cm}$. We did not detect any urethral stricture after a 6-month follow-up.
The stone composition was available in 12 patients of RPCCL, in which calcium oxalate (in 9 cases) was dominant.

\section{Discussion}

Although for many years the open approach was the gold standard and the most reliable form of therapy to remove large bladder stones, it has fallen into disfavor recently as a consequence of prolonged catheterization, increased length of hospital stays and a poor cosmetic outcome. ${ }^{13}$

The transurethral approach for bladder stone removal is an appealing technique as it gives the surgeon the opportunity to use the natural orifice to access; however, there are the limitations of the size $(>2 \mathrm{~cm})$ and the number of stones $(>4) .{ }^{14,15}$

The percutaneous technique is the preferred modality due to superior cosmetic results, less morbidity and a shorter hospital stay compared to the open procedure; in addition, there is not any stone size limitation in this technique versus the transurethral procedures. ${ }^{12,16,17}$

However, there are some challenges with the classic (antegrade) cystolitholapaxy. On the one hand, operative time in the management of bladder calculi is an important point because most of the patients in this field is aged (comprising prostatic enlargement) and in elderly people lengthened anesthesia is risky; furthermore, by rising the operation time, the irrigation and consequently the volume of liquid absorption would increase. On the other hand, classic bladder access is associated with the potential risk of dilatator over-advancement and damage to the posterior bladder wall and rectum during access to the bladder. ${ }^{18,19}$ Although the risk of injury can be reduced by simultaneous cystoscopy or fluoroscopy, they have their own limitations. Simultaneous cystoscopy needs another urologist and fluoroscopy encompasses the patient's and surgeon's exposure to radiation. ${ }^{19}$

Table 1. Demographic and Clinical Characteristics

\begin{tabular}{|c|c|c|c|c|c|}
\hline \multicolumn{2}{|l|}{ Variable } & All Case $(n=124)$ & RPCCL $(n=65)$ & TULL $(n=59)$ & $P$ Value \\
\hline \multicolumn{2}{|l|}{ AGE $(y)$} & $50.33 \pm 9.64$ & $49.65 \pm 11.12$ & $51.02 \pm 8.17$ & $\geq 0.05$ \\
\hline \multicolumn{2}{|l|}{ BMI } & $23.65 \pm 3.25$ & $23.2 \pm 3.43$ & $24.1 \pm 3.08$ & $\geq 0.05$ \\
\hline \multicolumn{2}{|l|}{ STONE SIZE (cm) } & $3.35 \pm 1.07$ & $3.66 \pm 0.94$ & $3.04 \pm 0.8$ & $\geq 0.05$ \\
\hline \multicolumn{2}{|l|}{ Number of stones } & $3.68 \pm 0.53$ & $4.33 \pm 1.05$ & $3.04 \pm 0.02$ & $\geq 0.05$ \\
\hline \multicolumn{2}{|l|}{ MOT (min) } & $29.15 \pm 7.12$ & $31.85 \pm 6.47$ & $47.30 \pm 4.27$ & $\leq 0.05$ \\
\hline \multicolumn{2}{|l|}{ MHS (day) } & $2.85 \pm 1.23$ & $2.6 \pm 0.67$ & $1.1 \pm 0.66$ & $\leq 0.05$ \\
\hline \multirow{4}{*}{ Stone Etiology $(\mathrm{N})$} & $\mathrm{SCl}$ & 57 & 33 & 24 & \multirow{4}{*}{ NA } \\
\hline & $\mathrm{BPH}$ & 23 & 10 & 13 & \\
\hline & UAB & 25 & 9 & 16 & \\
\hline & Others & 19 & 13 & 6 & \\
\hline \multirow{2}{*}{ SFR $(\%)$} & Immediate & 98.5 & 100 & 97 & $\geq 0.05$ \\
\hline & 1 month & 100 & 100 & 100 & $\geq 0.05$ \\
\hline
\end{tabular}

Abbreviation: PCCl, Percutaneous cystolithotomy; TULL, transurethral cystolithotripsy with holmium-YAG laser; BMI, Body mass Index; MOT, mean operative time; MHS, mean hospital stay; $\mathrm{SCl}$, spinal cord injury; $\mathrm{BPH}$, Benign prostatic hyperplasia that only consented to do the stone removal ; UAB, Underactive bladder; SFR, Stone free rate; NA. Not assessed. 
In the retrograde approach, there is not any chance of over-advancement since the dilatator enters the bladder under the control of the Benique, and also, no radiation or even cystoscopy is needed during the bladder access.

Endourologic options, namely percutaneous and transurethral, are favorable modalities for the management of bladder stones. Transurethral lithotripsy can be done by pneumatic, ultrasonic and laser lithotripter. ${ }^{20}$

We noted that the duration of surgery was reasonably short in retrograde assisted PCCL. Therefore, the risk of anesthesia and volume absorption would decrease consequently. This technique is also cost-benefit concerning a reduced amount of hospitalization time.

It is not mandatory to be an endourologist or a super professional urologist to do this method; furthermore, it does not need any unusual instruments and it can be done in every urology operating room even with limited facilities. ${ }^{21,22}$

\section{Conclusion}

This paper was an attempt to introduce a safe method which contains less complication, morbidity, and operation and hospital stay time and is incomparable with TULL. RPCCL is applicable in medical centers without Ho: YAG equipment.

\section{Limitation and Recommendation}

Clinical studies as a randomized clinical trial in both adult and pediatric age with larger patient series are necessary to confirm the potential advantages/disadvantage of this method.

\section{Ethical Considerations}

Researchers undertook to do the research based on the ethical committee of Shahid Beheshti and Kermanshah University of Medical Sciences. Written informed consent was obtained from all patients after explaining the risks, options and benefits of the method.

\section{Conflict of Interests}

The authors declare no conflict of interest.

\section{Acknowledgments}

The authors wish to acknowledge the assistance of Urology operation room staff.

\section{References}

1. Gupta DK, Mishra S. Percutaneous cystolithotripsy as a reliable modality to treat pediatric vesiculolithiasis. J Nep Med Col. 2017;14(1):18-20. doi:10.3126/jngmc. v14i1.17488

2. Kumar A, Dalela D, Goel A, Paul S, Sankhwar S. The twin amplatz sheath method: a modified technique of percutaneous cystolithotripsy for large bladder stones in female patients. J Surg tech Case Rep. 2013;5(2):109-11. doi: 10.4103/2006-8808.128757.

3. Razzaghi MR, Karkan MF, Ghiasy S, Javanmard B. Laser application in iran urology: a narrative review. J Lasers Med Sci. 2018;9(1):1-6. doi: 10.15171/jlms.2018.01.

4. Ahmadnia H, Kamalati A, Younesi M, Imani MM, Moradi M, Esmaeili M. Percutaneous treatment of bladder stones in children: 10 years experience, is blind access safe? Pediatr Surg Int. 2013;29(7):725-8. doi:10.1007/s00383013-3320-x

5. Javanmard B, Karkan MF, Razzaghi M, Ghiasy S, Ranjbar A, Rahavian A. Surgical management of vesical stones in children: a comparison between open cystolithotomy, percutaneous cystolithotomy and transurethral cystolithotripsy with holmium-YAG laser. J Lasers Med Sci. 2018;9(3):183-7. doi: 10.15171/jlms.2018.33.

6. Teichman JM, Rogenes VJ, McIver BJ, Harris JM. Holmium: yttrium-aluminum-garnet laser cystolithotripsy of large bladder calculi. Urol. 1997;50(1):44-8. doi: 10.1016/S00904295(97)00201-X

7. Karami H, Razaghi MR, Javanmard B, Yaghoob M, Hadad AH, Amani M, et al. Outpatient transurethral cystolithotripsy of large bladder stones by holmium laser. J Lasers Med Sci. 2016;7(1):12-15. doi: 10.15171/ jlms.2016.03.

8. Bodakci MN, Sancaktutar AA, Dağgulli M, Hatipoglu NK, Söylemez H, Penbegul N, et al. Micropercutaneous cystolithotomy in children: our experience with the transillumination technique. J Endourol. 2014;28(6):693-8. doi: 10.1089/end.2013.0636.

9. Tzortzis V, Aravantinos E, Karatzas A, Mitsogiannis IC, Moutzouris G, Melekos MD. Percutaneous suprapubic cystolithotripsy under local anesthesia. Urol. 2006;68(1):3841. doi: 10.1016/j.urology.2006.01.073

10. Liu G, Deng Y, Zhang S, Zhao X, Yao D, Zhang Q, et al. Minimally invasive percutaneous suprapubic cystolithotripsy: an effective treatment for bladder stones with urethral strictures. Int J Clin Exp Med. 2016;9(10):19907-12.

11. Torricelli FCM, Mazzucchi E, Danilovic A, Coelho RF, Srougi M. Surgical management of bladder stones: literature review. Rev Col Bras Cir. 2013;40(3):227-33.

12. Salati U, Lundeen C, Chew B, Buckley J. Percutaneous suprapubic cystolitholapaxy in adult patients with previous Mitrofanoff repair: considerations for the interventional radiologist. J Vasc Interv Radiol. 2017;28(9):1316-9. doi: 10.1016/j.jvir.2017.05.013.

13. Papatsoris AG, Varkarakis I, Dellis A, Deliveliotis C. Bladder lithiasis: from open surgery to lithotripsy. Urol Res. 2006;34(3):163. doi: 10.1007/s00240-006-0045-5

14. Bansal A, Kumar M, Sankhwar S, Goel S, Patodia M, Aeron $\mathrm{R}$, et al. Prospective randomized comparison of three endoscopic modalities used in treatment of bladder stones. Urologia. 2016;83(2):87-92. doi: 10.5301/uro.5000171

15. Kara C, Resorlu B, Cicekbilek I, Unsal A. Transurethral cystolithotripsy with holmium laser under local anesthesia in selected patients. Urology. 2009;74(5):1000-3. doi: 10.1016/j.urology.2009.05.095

16. Obaid AT. A comparative study of percutaneous suprapubic cystolitholapaxy versus open cystolithotomy in adults. Med J Babylon. 2013;10(1):11-24.

17. Wollin T, Singal R, Whelan T, Dicecco R, Razvi H, Denstedt J. Percutaneous suprapubic cystolithotripsy for treatment of large bladder calculi. J Endourol. 1999;13(10):739-44. 
doi: 10.1089/end.1999.13.739

18. Vasudevan V, Strine AC, Kaefer M. A novel technique for endoscopic management of stones in a continent urinary reservoir. Urology. 2014;83(6):1398-400. doi: 10.1016/j. urology.2014.02.012

19. Metwally AH, Sherief MH, Elkoushy MA. Safety and efficacy of cystoscopically guided percutaneous suprapubic cystolitholapaxy without fluoroscopic guidance. Arab J Urol. 2016;14(3):211-5. doi: 10.1016/j.aju.2016.05.001

20. Abedi AR, Razzaghi MR, Allameh F, Aliakbari F,
Fallahkarkan M, Ranjbar A. Pneumatic Lithotripsy Versus Laser Lithotripsy for Ureteral Stones. J Lasers Med Sci. 2018;9(4):233-6. doi:10.15171/jlms.2018.42

21. Gan W, Guo H, Yang R, Lian H, Yao L. Minimally invasive percutaneous cystolithotomy: an effective treatment for bladder stones in infants aged $<1$ year. BJU Int. 2010;106(2):275-7. doi: 10.1111/j.1464-410X.2009.08995.x

22. Paez E, Reay E, Murthy L, Pickard RS, Thomas DJ. Percutaneous treatment of calculi in reconstructed bladder. J Endourol. 2007;21(3):334-6. doi: 10.1089/end.2006.0251 